TRANSACTIONS OF THE

AMERICAN MATHEMATICAL SOCIETY

Volume 360, Number 10, October 2008, Pages 5155-5172

S 0002-9947(08)04500-5

Article electronically published on May 27, 2008

\title{
ASYMPTOTIC BEHAVIOUR OF CODIMENSIONS OF P. I. ALGEBRAS SATISFYING CAPELLI IDENTITIES
}

\author{
ALLAN BERELE AND AMITAI REGEV
}

\begin{abstract}
Let $A$ be a p. i. algebra with 1 in characteristic zero, satisfying a Capelli identity. Then the cocharacter sequence $c_{n}(A)$ is asymptotic to a function of the form $a n^{g} \ell^{n}$, where $\ell \in \mathbb{N}$ and $g \in \mathbb{Z}$.
\end{abstract}

The second author conjectured that if $A$ is any p. i. algebra in characteristic zero, with cocharacter sequence $c_{n}(A)$, then the asymptotic behaviour of this sequence should be given by

$$
c_{n}(A) \simeq a \cdot n^{t} \cdot \ell^{n}
$$

where $\ell$ is a non-negative integer, $t$ is an integer or a half-integer, and $a$ belongs to $\mathbb{Q}[\sqrt{2 \pi}, \sqrt{b}]$, for some $0<b \in \mathbb{Z}$. We call $\ell^{n}$ the exponential part and $a \cdot n^{t}$ the rational part of the codimension growth.

Giambruno and Zaicev made progress on this conjecture by proving that for some non-negative integer $\ell$,

$$
f_{1}(n) \ell^{n} \leq c_{n}(A) \leq f_{2}(n) \ell^{n},
$$

where $f_{1}(n)$ and $f_{2}(n)$ are Laurent polynomials, but not necessarily of the same degree.

In this paper we make some progress towards verifying the above conjecture by proving the following theorem.

Theorem 1. Let $A$ be a p.i. algebra with 1 satisfying a Capelli identity. Then

$$
c_{n}(A) \simeq a \cdot n^{g} \cdot \ell^{n}
$$

where, furthermore, $g \in \frac{1}{2} \mathbb{Z}$. Also, the constant a here is a sum of Selberg-type integrals; see for example Theorem 36 below.

This theorem can help to determine whether or not the generating function $f(x)=\sum_{n \geq 0} c_{n}(A) x^{n}$ of the codimensions is algebraic. For example, if $g \in \mathbb{Z}$ and is negative, then $f(x)$ is not algebraic; see Lemma 3.2 in [1.

This application raises the following further question about Theorem 1] when is $g<0$ ? The techniques of [6] imply the following: Let $A_{j}, j=1,2$, be p. i. algebras with $T$-ideals of identities $i d\left(A_{j}\right)=I_{j}$. This is known to be the case, for example,

Received by the editors June 5, 2006.

2000 Mathematics Subject Classification. Primary 16R10.

Key words and phrases. Polynomial identities, cocharacter sequence.

The work of the first author was supported by both the Faculty Research Council of DePaul University and the National Security Agency, under Grant MDA904-500270. The United States Government is authorized to reproduce and distribute reprints notwithstanding any copyright notation herein.

The work of the second author was partially supported by ISF grant 947-04.

(C)2008 American Mathematical Society 
if $A_{j}$ is the algebra of $n \times n$ matrices. Assume $c_{n}\left(A_{i}\right) \simeq a_{j} \cdot n^{g_{j}} \cdot \ell_{j}^{n}$ with $g_{1}, g_{2}<0$. Then the algebras with $T$-ideals of identities equal to $I_{1} I_{2}$ and $I_{1} \cap I_{2}$ also have that same property $g<0$. However, the comment at the end of the introduction implies that one can construct many algebras with $g \geq 0$.

The Giambruno-Zaicev theorem was proven in two stages: In [10] they proved it in the special case that $A$ is finitely generated, or more generally that $A$ satisfies a Capelli identity, and in [11] they proved the general case. In the current paper we will prove a slightly weaker version of the conjecture in the case that $A$ satisfies a Capelli identity and has a unit. We will show that $c_{n}(A) \simeq a n^{t} \ell^{n}$, where $\ell$ is a non-negative integer, $t$ is an integer or half integer but we don't have control over the constant $a$. Getting such control would involve evaluating a certain integral, which we describe in the second section. Under the weaker assumption that $c_{n}(A)$ is an increasing sequence, we prove that $a_{1} n^{t} \ell^{n} \leq c_{n}(A) \leq a_{2} n^{t} \ell^{n}$.

The proof combines and adapts ideas from the first author's paper [5] and the second author's paper [14. The main idea is that for any p. i. algebra, $c_{n}(A)=$ $\sum m_{\lambda} d_{\lambda}$, where $d_{\lambda}$ is the degree of the $S_{n}$-character corresponding to $\lambda$, and $m_{\lambda}$ is the multiplicity of that character in the cocharacter sequence. If $A$ satisfies a Capelli identity, then $m_{\lambda}$ will be zero unless $\lambda$ has height bounded by some constant $k$. For such a $\lambda$, the multiplicities were estimated in [5]. We think of the multiplicities as functions from $\mathbb{N}^{k} \rightarrow \mathbb{N}$. In $\left[5\right.$ we showed that $\mathbb{N}^{k}$ could be partitioned into a finite number of regions such that $m_{\lambda}$ was equal to a polynomial on each. In section 1 we show that as far as the asymptotic behaviour of $c_{n}$ is concerned, we need only consider regions of the form

$$
\left\{v_{0}+\alpha_{1} v_{1}+\cdots+\alpha_{r} v_{r} \mid \alpha_{1}, \ldots, \alpha_{r} \geq 0\right\} \cap\left(\underline{i}+(d \mathbb{Z})^{k}\right),
$$

where the $v_{i}$ are all partitions, all except $v_{0}$ are linearly independent and have height at most the exponent $\ell \leq k$, and $v_{1}$ is the partition $\left(1^{\ell}\right)$, thought of as the vector $(1, \ldots, 1,0, \ldots, 0)$ ( $\ell$ 1's and $k-\ell 0$ 's). In the next section, we compute $\sum m_{\lambda} d_{\lambda}$ over one such region, adapting the arguments from [14. In that work the second author computes the asymptotic behaviour of $\sum d_{\lambda}^{\beta}$, where $\lambda$ runs over partitions of $n$ with at most $\ell$ parts. The sum we need to compute differs both in the region we are summing over and the function we are summing, but for all that, the computations from [14 turn out to be just what we need here. The main result of this section is that $\sum m_{\lambda} d_{\lambda}$ over such a domain is asymptotic to a function of the form $a n^{t} \ell^{n}$, where $a, t$ and $\ell$ all have the required form. Finally, in the last section we deal with the technical problems involved in summing the $\sum m_{\lambda} d_{\lambda}$ from the various domains.

Based on these computations we present a conjecture.

Conjecture. Let $f\left(x_{1}, \ldots, x_{k}\right) / g\left(x_{1}, \ldots, x_{k}\right)$ be a rational function such that the numerator and denominator are each symmetric polynomials with integer coefficients, and such that the denominator is a product of terms of the form $\left(1-x_{1}^{a_{1}} \cdots x_{k}^{a_{k}}\right)$. We expand the fraction as a series in Schur functions,

$$
f\left(x_{1}, \ldots, x_{k}\right) / g\left(x_{1}, \ldots, x_{k}\right)=\sum_{n=0}^{\infty} \sum_{\lambda \in \operatorname{Par}(n)} m_{\lambda} S_{\lambda}\left(x_{1}, \ldots, x_{k}\right)
$$

so that $m_{\lambda}$ is the coefficient of the Schur function $S_{\lambda}\left(x_{1}, \ldots, x_{k}\right)$. Define $c_{n}$ to be $\sum_{\lambda \in \operatorname{Par}(n)} m_{\lambda} d_{\lambda}$. Then there exists a modulus $d$ and constants $a_{i}, t_{i}$ and $\ell_{i}$ where 
$i=0, \ldots, d-1$ such that, if we restrict $n$ to be congruent to $i \bmod d$, then $c_{n}$ has asymptotic behaviour

$$
c_{n} \simeq a_{i} n^{t_{i}} \ell_{i}^{n} .
$$

Moreover, each $t_{i}$ will be either an integer or a half-integer and each $\ell_{i}$ will be the $d^{\text {th }}$-root of a rational number.

We close this introduction with a lower bound on the degree of the rational part of $c_{n}(A)$.

Theorem 2. Let $A$ be a $p$. $i$. algebra satisfying a Capelli identity with $c_{n}(A) \simeq$ $a n^{t} \ell^{n}$. Let $1 \leq s \leq 4$ be minimal such that $\ell$ can be written as a sum of $s$ squares. Then $t \geq \frac{3}{2} s-1-\frac{1}{2} \ell$.

Proof. Let $I$ be the ideal of identities of $A$, and denote by $I_{a}$ the ideal of identities of $a \times a$ matrices. It is implicit in [10] and explicit in Theorems 2.5 and 2.8 of [7] that there exist $a_{1}, \ldots, a_{k}$ such that $I \subseteq I_{a_{1}} \cdots I_{a_{k}}$ and such that the generic algebra with identities $I_{a_{1}} \cdots I_{a_{k}}$ has the same exponential rate of growth as $A$. Since this algebra will have cocharacter sequence less than or equal to that of $A$, it suffices to prove our lower bound in the case of $I=I_{a_{1}} \cdots I_{a_{k}}$. It follows from Theorem 1.4 of [6] that the exponential rate of growth of such an algebra is $\ell=a_{1}^{2}+\cdots+a_{k}^{2}$ and that the degree of the rational part is

$$
t=k-1+\sum-\frac{1}{2}\left(a_{i}^{2}-1\right)=\frac{3}{2} k-1-\frac{1}{2} \ell .
$$

We minimize this sum by making $k$ as small as possible.

It turns out that there is no upper bound on the degree of the rational part. Here is an example taken from [13]. It generalizes easily to any value of $\ell$. Let $A$ be the subalgebra of $k \times k$ matrices spanned by $e_{11}$ and all $e_{i j}$ with $i<j$. Then all identities of $A$ are consequences of $\left[x_{1}, x_{2}\right] x_{3} \cdots x_{k}$ and $c_{n}(A) \simeq a n^{k-1}$. So, the exponent $k-1$ can be made as large as desired while the exponential rate of growth is equal to 1 .

\section{The Multiplicities $m_{\lambda}$}

We first describe the relevant theorem from $[5]$. The space $\mathbb{R}^{k}$ can be partitioned into a finite number of subsets such that the multiplicity function $m_{\lambda}$ restricted to each of these subsets is equal to a polynomial in the coordinates. In order to describe this decomposition, it is useful to have a definition.

Definition 3. Given $v_{1}, \ldots, v_{r}$ vectors in $\mathbb{R}^{k}$ the linear cone $C\left(v_{1}, \ldots, v_{r}\right)$ is defined by

$$
C\left(v_{1}, \ldots, v_{r}\right)=\left\{\alpha_{1} v_{1}+\cdots+\alpha_{r} v_{r} \mid \alpha_{1}, \ldots, \alpha_{r} \geq 0\right\} .
$$

Moreover, given an additional vector $v_{0}$ the affine linear cone $C\left(v_{0} ; v_{1}, \ldots, v_{r}\right)$ is defined to be $v_{0}+C\left(v_{1}, \ldots, v_{r}\right)$. This is a translation of $C\left(v_{1}, \ldots, v_{r}\right)$ by the vector $v_{0}$. If in addition the vectors $v_{1}, \ldots, v_{r}$ are linearly independent we call $C\left(v_{1}, \ldots, v_{r}\right)$ a simplicial cone and $C\left(v_{0} ; v_{1}, \ldots, v_{r}\right)$ a simplicial affine cone, and we call $v_{1}, \ldots, v_{r}$ the basis in either case. Finally, if the $v_{i}$ can all be taken to have coordinates in $\mathbb{Q}$ we call the resulting cone rational.

Lemma 4. Any (rational or affine) cone is a finite union of (rational or affine) simplicial cones with disjoint interiors. 
Proof. Let $d$ be the dimension of the span of $v_{1}, \ldots, v_{r}$. We use induction on $d$. If $d=1$, then the cone is a ray and so is already simplicial. If $d>1$, then the boundary of $C$ is a union of $(d-1)$-dimensional cones, so it is a finite union of simplicial cones $v_{0}+C_{1}, \ldots, v_{0}+C_{m}$. Let $v$ be a vector with rational coordinates such that $v_{0}+v$ is in the interior of $C$. Then each of the $C_{i}$ together with $v$ span a simplicial cone $C_{i}^{\prime}$. Since the $C_{i}$ have disjoint interiors, so do the $C_{i}^{\prime}$; and since $C$ is convex, the union of the $C_{i}^{\prime}$ gives all of $C$.

Here then is the theorem we need from [5].

Theorem 5. Given a p. i. algebra A with cocharacter supported in the strip of height $k$, there exists a positive integer $d$ and a partition of $\left(\mathbb{R}^{+}\right)^{k}$ into regions $R_{1}, \ldots, R_{m}$, each a finite intersection of rational affine linear cones such that $m_{\lambda}$ is given by a polynomial on each $R_{a} \cap\left(\underline{i}+(d \mathbb{Z})^{k}\right)$, where $\underline{i} \in\{0, \ldots, d-1\}^{k}$.

Note that here and throughout we use $\mathbb{R}^{+}$instead of $\mathbb{R}^{\geq 0}$ for the non-negative real numbers.

Here is an example to illustrate the theorem; see [4] and 9]. It involves trace cocharacters instead of ordinary cocharacters, but the theory is the same.

Theorem. Let $\lambda=\left(\lambda_{1}, \lambda_{2}\right)$ be a partition of height at most 2 . Then the multiplicity of $\chi^{\lambda}$ in the trace cocharacter sequence of $3 \times 3$ matrices depends on whether $\lambda_{1}>$ $2 \lambda_{2}$. If this is the case, then

$$
m_{\lambda}=\frac{\lambda_{1}^{2} \lambda_{2}^{5}}{17280}-\frac{11 \lambda_{1} \lambda_{2}^{6}}{103680}+\frac{71 \lambda_{2}^{7}}{1451520}+\mathcal{O}\left(n^{6}\right)
$$

and if not, then

$m_{\lambda}=\frac{\lambda_{1}^{7}}{1451520}-\frac{\lambda_{1}^{6} \lambda_{2}}{103680}+\frac{\lambda_{1}^{5} \lambda_{2}^{2}}{17280}-\frac{\lambda_{1}^{4} \lambda_{2}^{3}}{5184}+\frac{\lambda_{1}^{3} \lambda_{2}^{4}}{2592}-\frac{7 \lambda_{1}^{2} \lambda_{2}^{5}}{17280}+\frac{7 \lambda_{1} \lambda_{2}^{6}}{34560}-\frac{19 \lambda_{2}^{7}}{483840}+\mathcal{O}\left(n^{6}\right)$. Moreover, each of the two $\mathcal{O}\left(n^{6}\right)$ terms is given by 36 (not necessarily distinct) polynomials, depending on $\lambda_{1}$ and $\lambda_{2} \bmod 6$.

Note that the set of partitions is divided into partitions with $\lambda_{1} \geq 2 \lambda_{2}$ and $\lambda_{1} \leq 2 \lambda_{2}$. These two sets are each cones: The former is $C((2,1),(1,0))$ and the latter is $C((2,1),(1,1))$, and within each of these cones, the partitions are further divided up according to which lattice $(a, b)+6 \mathbb{Z} \times 6 \mathbb{Z}$ they belong to.

Definition 6. Given a real function $f$ on $D \subseteq \mathbb{Z}^{k}$ such that there exists a modulus $d$ and such that $f$ restricted to each $D \cap(\underline{i}+(\bar{d} \mathbb{Z}))^{k}$ is equal to a polynomial function for each $\underline{i} \in\{0, \ldots, d-1\}^{k}$, we will say that $f$ is almost polynomial.

In Theorem 5, each $R_{i}$ is a finite intersection of affine cones. Our next goal is to show that each such intersection can be written as a finite union of simplicial cones.

Definition 7. A polyhedral set in $\mathbb{R}^{k}$ is an intersection of finitely many halfspaces. If the half-spaces can all be defined by equations with rational coefficients, we call the polyhedral set rational. Note that the intersection of the half-spaces $a_{1} x_{1}+\cdots+a_{k} x_{k} \geq b$ and $a_{1} x_{1}+\cdots+a_{k} x_{k} \leq b$ is an $(n-1)$-dimensional hyperplane, and so a polyhedral set may be contained in a hyperplane of dimension less than $k$. The dimension of a polyhedral set is the dimension of the smallest hyperplane containing it. (We use the term hyperplane to refer to a translate of a subspace of $\mathbb{R}^{k}$, not necessarily of dimension $k-1$.) Note that an intersection of affine cones 


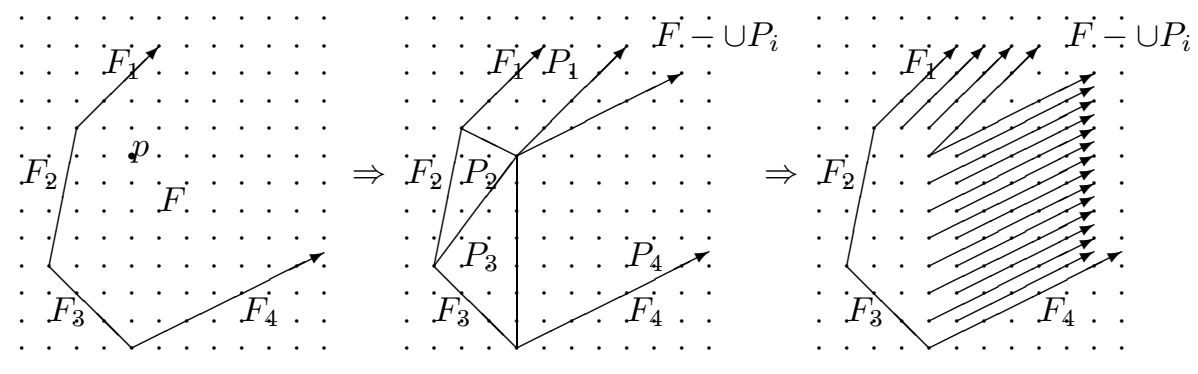

Figure 1. Proof of Lemma 10

is a polyhedral set. Given $S \subseteq \mathbb{R}^{k}$ we denote by $\operatorname{conv}(S)$ the convex closure of $S$, i.e., the smallest convex set containing $S$.

Lemma 8. Let $F \subseteq \mathbb{R}^{k}$ be a d-dimensional rational polyhedral set contained in the d-dimensional hyperplane $\Pi$ and $p \in \mathbb{R}^{k}-\Pi$. Then there exist finitely many disjoint rational polyhedral sets each with the same dimension as $F$ and together containing all of the integer points of conv $(p, F)$.

Proof. Let $\Pi^{\prime}$ be a $(k-1)$-dimensional rational hyperplane containing $\Pi$ and not containing $p$. Let $\Pi^{\prime}$ have equation $a_{1} x_{1}+\cdots+a_{k} x_{k}=b$, where the $a_{i}$ and $b$ are all integers and the $a_{i}$ are relatively prime. Let $p=\left(p_{1}, \ldots, p_{k}\right)$ and let $a_{1} p_{1}+\cdots+$ $a_{k} p_{k}=b^{\prime}$. Note that $\operatorname{conv}(p, F)$ is contained between these two hyperplanes. But there are only finitely many integers $b_{j}$ between $b$ and $b^{\prime}$ (inclusive) and so only finitely many hyperplanes parallel to $\Pi^{\prime}$ containing integer points. Each of these hyperplanes intersects $\operatorname{conv}(F, p)$ in a polyhedral set having the same dimension as $F$.

We now need a general fact from convex set theory. Let $S \subseteq \mathbb{R}^{k}$ be any infinite, closed, convex set, and let $p$ and $q$ be two points of $S$. Given a $v \in \mathbb{R}^{k}$ the ray $p+\mathbb{R}^{+} v$ will be contained in $S$ if and only if the ray $q+\mathbb{R}^{+} v$ is contained in $S$. Based on this fact, the characteristic cone of $S$ is defined to be

$$
\operatorname{cc}(S)=\left\{v \mid p+\mathbb{R}^{+} v \subseteq S\right\} .
$$

An important theorem about $\operatorname{cc}(S)$ is that it is indeed a cone and if $S$ is a polyhedral set, then it will be a linear cone; see for example section 2.5 of [12] or section 1.5 of [16. We record this as a lemma.

Lemma 9. Let $S \subseteq \mathbb{R}^{k}$ be any infinite, closed, convex set, and let $p$ be a point of $S$. Then the set of rays with endpoint $p$ which are completely contained in $S$ forms a cone.

Lemma 10. Let $F$ be a rational polyhedral set in $\mathbb{R}^{k}$. Then there exist finitely many rational affine linear cones whose interiors are disjoint and whose union contains all but finitely many of the integer points of $F$.

Proof. We use induction on $d=\operatorname{dim} F$ to prove the lemma. The case of $d=1$ is easy since a convex one-dimensional set is a line segment or a ray. A line segment will contain only finitely many integer points, and a ray is an affine linear cone.

In the general case, let $F$ be a rational polyhedral set of dimension $d$ and let $p$ be a rational point in the interior of $F$; see Figure 1 . The $(d-1)$-dimensional 
faces of $F$ are called facets and their union is the boundary of $F$. Let $F_{1}, \ldots, F_{a}$ be the facets of $F$ and for each $i$ let $P_{i}=\operatorname{conv}\left(F_{i}, p\right)$. For example, in Figure 1, the two-dimensional figure $F$ is bounded by the one-dimensional facets $F_{1}, \ldots, F_{4}$. Note that if the facet $F_{i}$ is contained by the $(d-1)$-dimensional hyperplane $\Pi_{i}$, then $p \in \mathbb{R}^{k}-\Pi_{i}$; hence Lemma 8 can be applied. By that lemma, the integer points of $P_{i}$ are covered by finitely many $(d-1)$-dimensional rational polyhedral sets. So there exist polyhedral sets of dimension $d-1, P_{i j}$, such that $\cup_{j} P_{i j}$ has the same integer points as $P_{i}$. By induction each $P_{i j}$ is the union of disjoint rational affine cones. In the example, $P_{2}$ and $P_{3}$ are finite and will have only finitely many integer points, and these points are not covered by cones in this example. The regions $P_{1}$ and $P_{4}$ are infinite, and Lemma 8 implies that in each there are a finite number of rays containing all of their rational points.

However, the union of the $P_{i}$ may not cover all of $F$. The points of $F-\cup P_{i}$ consist of the union of all rays with endpoint $p$ which do not intersect the boundary of $F$. Hence it is the union of all rays starting at $p$ and completely contained in $F$. In the example, $F-\cup P_{i}$ is a 2-dimensional cone. In general, we may apply Lemma 9 to conclude that it is an affine cone. Hence $F$ will be the union of this cone with the $P_{i}$, and since there are finitely many affine cones containing all but finitely many of the integer points of $\cup P_{i}$, this completes the proof.

Corollary 11. Let $F$ be a rational polyhedral set in $\mathbb{R}^{k}$. Then there exist finitely many disjoint rational, affine simplicial cones whose union contains all but finitely many of the integer points of $R$.

Proof. In Lemma 10 if two of the cones intersect along their boundary we replace one by a slightly smaller cone and then use Lemma 4 to replace the cones by simplicial ones.

In light of this corollary, we may now reformulate Theorem 5 .

Corollary 12. Given a p. i. algebra A with cocharacter supported in the strip of height $k$, there exist a positive integer $d$ and a partition of $\left(\mathbb{R}^{+}\right)^{k}$ into disjoint rational, affine simplicial cones $C_{1}, \ldots, C_{m}$ and a finite number of points such that $m_{\lambda}$ is given by a polynomial on each $C_{a} \cap\left(\underline{i}+(d \mathbb{Z})^{k}\right)$, where $\underline{i} \in\{0, \ldots, d-1\}^{k}$.

One of the main results of [14] is that if $\lambda$ has all parts close to equal, then $d_{\lambda}$ has exponential behaviour $k^{n}$. Lemma 16 below shows that if none of the basis vectors is $(1, \ldots, 1)$, then the exponential behaviour of $d_{\lambda}$ is strictly less.

Remark 13. Given $v \in \mathbb{R}^{k}$, denote $v=\left(v^{(1)}, \ldots, v^{(k)}\right)$. It is a partition if $v \in \mathbb{Z}^{k}$ and $v^{(1)} \geq \cdots \geq v^{(k)} \geq 0$. Given $u=\left(u^{(1)}, \ldots, u^{(k)}\right) \in \mathbb{R}^{k}$ with $u^{(1)}, \ldots, u^{(k)} \geq 0$, denote $|u|=u^{(1)}+\cdots+u^{(k)}$. Let $C=C\left(v_{0} ; v_{1}, \ldots, v_{r}\right)$ be a cone, (w.l.o.g.) $v_{1}, \ldots, v_{r} \neq 0$, and let $u=\left(u^{(1)}, \ldots, u^{(k)}\right) \in C$, so $u=v_{0}+\sum_{i=1}^{r} t_{i} v_{i}$; if $v_{0}, \ldots, v_{r}$ are partitions, then $u^{(1)} \geq \cdots \geq u^{(k)} \geq 0$ and $|u|=\left|v_{0}\right|+\sum_{i=1}^{r} t_{i}\left|v_{i}\right|$. Moreover, since $v_{1}, \ldots, v_{r} \neq 0,|u| \rightarrow \infty$ whenever $\sum_{i} t_{i} \rightarrow \infty$. If the partition $v_{i}$ is not proportional to $(1, \ldots, 1)$, then there exist $0 \leq \delta_{i}<1$ such that $v_{i}^{(k)} \leq\left(\delta_{i} / k\right)\left|v_{i}\right|$.

Lemma 14. Let $C=C\left(v_{0} ; v_{1}, \ldots, v_{r}\right) \subseteq \mathbb{R}^{k}$ be a cone, $v_{1}, \ldots, v_{r} \neq 0$ partitions where none is proportional to $(1, \ldots, 1)$. There exist $0 \leq \delta<1$ such that for all $u=\left(u^{(1)}, \ldots, u^{(k)}\right)=v_{0}+\sum_{i=1}^{r} t_{i} v_{i} \in C, u^{(k)} \leq(\delta / k)|u|$, provided that $\sum_{i} t_{i}$ is large enough. 
Proof. Write $v_{i}=\left(v_{i}^{(1)}, \ldots, v_{i}^{(k)}\right)$, so $\left|v_{i}\right|=v_{i}^{(1)}+\cdots+v_{i}^{(k)}, i=1, \ldots, r$. For each $i$ there exists $0 \leq \delta_{i}<1$ such that $v_{i}^{(k)} \leq\left(\delta_{i} / k\right)\left|v_{i}\right|$.

Let $\delta^{\prime}=\max \left\{\delta_{i} \mid i=1, \ldots, r\right\}$, so $0 \leq \delta^{\prime}<1$. Choose some $\delta^{\prime}<\delta<1$, for example $\delta=\left(\delta^{\prime}+1\right) / 2$. Now let $u=\left(u^{(1)}, \ldots, u^{(k)}\right)=v_{0}+\sum_{i=1}^{r} t_{i} v_{i} \in C$. Then

$$
u^{(k)}=v_{0}^{(k)}+\sum_{i=1}^{r} t_{i} v_{i}^{(k)} \leq v_{0}^{(k)}+\sum_{i=1}^{r} t_{i} \frac{\delta_{i}}{k}\left|v_{i}\right| \leq v_{0}^{(k)}+\frac{\delta^{\prime}}{k} \sum_{i=1}^{r} t_{i}\left|v_{i}\right|=v_{0}^{(k)}+\frac{\delta^{\prime}}{k}|u| .
$$

If $\sum_{i=1}^{r} t_{i}$ is large, then also $\sum_{i=1}^{r} t_{i}\left|v_{i}\right|$ becomes large and $v_{0}^{(k)}$ is negligible, so $v_{0}^{(k)}+\frac{\delta^{\prime}}{k}|u| \leq \frac{\delta}{k}|u|$. Therefore $u^{(k)} \leq \frac{\delta}{k}|u|$.

Lemma 15. Let $0 \leq \delta<1$ and consider partitions $\lambda=\left(\lambda_{1}, \ldots, \lambda_{k}\right)$ satisfying $\lambda_{k} \leq(\delta / k)|\lambda|$. Then, as $|\lambda| \rightarrow \infty$, the exponential growth of $d_{\lambda}\left(=f^{\lambda}\right)$ is bounded by $(k-\epsilon)^{|\lambda|}$ for some $\epsilon>0$.

Proof. Let $n=|\lambda|$. By the hook formula,

$$
d_{\lambda}=\frac{n !}{\prod h_{i j}} \leq \frac{n !}{\lambda_{1} ! \cdots \lambda_{k} !} .
$$

Write $\lambda_{k}=\left(\frac{1}{k}-x\right) \cdot n$, so $0<(1-\delta) / k \leq x$. Clearly, $\lambda_{1}+\cdots+\lambda_{k-1}=n-\lambda_{k}=$ $\left(\frac{k-1}{k}+x\right) \cdot n$. Fixing $\lambda_{k}$, it is well known that the product $\lambda_{1} ! \cdots \lambda_{k-1}$ ! minimizes when $\lambda_{1} \approx \cdots \approx \lambda_{k-1}$, so when $\lambda_{1} \approx \cdots \approx \lambda_{k-1} \approx\left(\frac{1}{k}+\frac{x}{k-1}\right) \cdot n$, in which case $\frac{n !}{\prod h_{i j}} \leq \frac{n !}{\lambda_{1} ! \cdots \lambda_{k} !}$ maximizes. Hence, by (1),

$$
\begin{gathered}
d_{\lambda} \leq \frac{n !}{\lambda_{1} ! \cdots \lambda_{k} !} \\
\lambda_{1} \approx \cdots \approx \lambda_{k-1} \approx\left(\frac{1}{k}+\frac{x}{k-1}\right) \cdot n, \quad \lambda_{k}=\left(\frac{1}{k}-x\right) \cdot n .
\end{gathered}
$$

Apply Stirling's formula $m ! \approx \sqrt{2 \pi} \cdot e^{-m} m^{m} \sqrt{m}$ to the r.h.s. of (2) $)$. After discarding a few terms of the order of magnitude of $\sqrt{n}$ we have $d_{\lambda} \lesssim(1 / g(x))^{n}$ where

$$
g(x)=\left(\frac{1}{k}+\frac{x}{k-1}\right)^{(k-1)\left(\frac{1}{k}+\frac{x}{k-1}\right)}\left(\frac{1}{k}-x\right)^{\left(\frac{1}{k}-x\right)} .
$$

It is easy to show that $g(x)$ monotonically increases: let $h(x)=\ln g(x)$; then $h^{\prime}(x)=\ln \left[\left(\frac{1}{k}+\frac{x}{k-1}\right) /\left(\frac{1}{k}-x\right)\right]>0$. Thus $1 / g(x)$ decreases with $x$. Since $g(0)=1 / k$, therefore for $0<x \leq 1 / k, g(x)>1 / k$ and we can write $1 / g(x)=k-\epsilon$ for some $\epsilon>0$.

As a corollary of Lemmas 14 and 15 we now have

Lemma 16. Let $C=C\left(v_{0} ; v_{1}, \ldots, v_{r}\right) \subseteq \mathbb{R}^{k}$ be an affine linear cone in which the basis vectors are all partitions and none is a multiple of $(1, \ldots, 1)$. Then there exists an $\epsilon>0$ and a polynomial $p\left(x_{1}, \ldots, x_{k}\right)$ such that for every partition $\lambda \in C$, $d_{\lambda} \leq p(\lambda)(k-\epsilon)^{|\lambda|}$.

Given $0 \leq \ell \leq k$ we let $\mathbb{R}^{k, \ell} \subseteq \mathbb{R}^{k}$ be the vectors with the last $k-\ell$ coordinates all equal to zero and we let $1_{k, \ell}$ be the vector in $\mathbb{R}^{k, \ell}$ with first $\ell$ coordinates equal to 1 . We now have this corollary. 
Corollary 17. Let $C=C\left(v_{0} ; v_{1}, \ldots, v_{r}\right)$ be an affine linear cone in $\mathbb{R}^{k}$ in which $v_{1}, \ldots, v_{r} \in \mathbb{R}^{k, \ell}$ and none of $v_{1}, \ldots, v_{r}$ is a multiple of $1_{k, \ell}$. Then there exists an $\epsilon>0$ and a polynomial $p\left(x_{1}, \ldots, x_{k}\right)$ such that for every partition $\lambda \in C$, $d_{\lambda} \leq p(\lambda)(\ell-\epsilon)^{|\lambda|}$.

In [10] Giambruno and Zaicev proved that for any p. i. algebra $A$ satisfying a Capelli identity of degree $k$, the sequence $\left\{\sqrt[n]{c_{n}(A)}\right\}$ has a limit $\ell=e(A)$ which is a non-negative integer less than or equal to $k$. (Gaimbruno and Zaicev denote the limit by $e$ for exponential rate of growth, but we need to reserve $e$ for $2.71 \cdots$.) In the course of proving this (Theorem 3 in their paper), they also proved that there was a constant $K$ (in their paper $K=d q-d$ ) such that for every $n$, the cocharacter sequence contains $\chi^{\lambda}, \lambda \in \operatorname{Par}(n)$, with non-zero multiplicity and $\lambda$ close to a rectangle of height $\ell$, in the sense that $\sum_{i=1}^{\ell}\left|\lambda_{i}-\frac{n}{\ell}\right| \leq K$, and $\sum_{i>\ell} \lambda_{i} \leq K$, and that it doesn't contain any $\chi^{\lambda}$ with $\lambda=\left(\lambda_{1}, \lambda_{2}, \ldots\right)$ and with $\lambda_{\ell+1}$ large. Translating these two facts into the language of Theorem 5 yields the following lemma. We attribute it to Giambruno and Zaicev since it is immediate from their work. To state the lemma more easily, let $C=C\left(v_{0} ; v_{1}, \ldots, v_{r}\right)$ be a simplicial cone. We will say that $C$ is in the support of $m_{\lambda}$ if $m_{\lambda}$ is a non-zero polynomial on the intersection of $C$ with some lattice $\underline{i}=(d \mathbb{Z})^{k}$.

Lemma 18 (Giambruno and Zaicev). Let $A$ be a p. i. algebra which satisfies a Capelli identity and with exponential rate of growth $\ell$ of the codimensions. Let $C$ be a simplicial cone in the support of $m_{\lambda}$, as above. Then each of the basis elements of $C$ is a partition of height at most $\ell$. Moreover, there exists at least one simplicial cone $C$ in the support of $m_{\lambda}$ with basis element $\left(1^{\ell}\right)$.

Proof. Let $C=C\left(v_{0} ; v_{1}, \ldots, v_{t}\right)$ be a simplicial cone in the support of $m_{\lambda}$. So, on the intersection of $C$ and a lattice, the multiplicity function equals a non-zero polynomial $p\left(\lambda_{1}, \ldots, \lambda_{k}\right)$. Let $q\left(\alpha_{1}, \ldots, \alpha_{t}\right)$ be the multiplicity of $\lambda=\alpha_{1} v_{1}+$ $\cdots+\alpha_{t} v_{t}$. Note that each $\lambda_{j}$ equals $\sum_{i} \alpha_{i} v_{j, i}$, where $v_{j, i}$ is the $i^{\text {th }}$ coordinate of $v_{j}$, and so $q$ is obtained from $p$ by a linear substitution. Hence $q$ is also a non-zero polynomial. Assume by way of contradiction that $v_{1}$ is a partition of height $\geq \ell+1$. Write $q$ as $\sum_{i} \alpha_{1}^{i} q_{i}\left(\alpha_{2}, \ldots, \alpha_{t}\right)$. For any choice of $\alpha_{2}, \ldots, \alpha_{t}$ in the lattice, there would exist infinitely many values of $\alpha_{1}$ in the lattice such that $v_{0}+\alpha_{1} v_{1}+\cdots+\alpha_{t} v_{t}$ is not a partition with $\lambda_{\ell+1}$ small. By Giambruno and Zaicev's theorem, the multiplicity $m_{\lambda}$ would be zero in these cases. Hence, by a Vandermonde argument each $q_{i}$ would be zero. This would imply that $m_{\lambda}$ is identically zero.

On the other hand, if there were no simplicial cones with $\left(1^{\ell}\right)$ as a basis element, then the exponential rate of growth would be less than $\ell$, by Corollary 17 .

Theorem 19. Let $A$ be a $p$. i. algebra which satisfies a Capelli identity of degree $k$ such that $e(A)=\ell$. Then there exists a set of affine simplicial cones $C_{1}, \ldots, C_{a} \subseteq$ $\mathbb{R}^{k}$, each with $1_{k, \ell}$ as a basis vector and with all basis vector partitions in $\mathbb{R}^{k, \ell}$ such that

$$
c_{n}(A) \simeq \sum_{i=1}^{a}\left\{m_{\lambda} d_{\lambda} \mid \lambda \in \operatorname{Par}(n) \cap C_{i}\right\}
$$

and such that the multiplicity function $m_{\lambda}$ restricted to each $C_{i}$ is almost polynomial. 
Proof. Combining Theorem 5 with Corollary 11] shows that $\operatorname{Par}(n)$ can be written as a union of a finite number of disjoint rational affine simplicial cones $\left\{C_{i}^{\prime}\right\}$ containing all partitions of height at most $k$ such that $m_{\lambda}$ is almost polynomial in each one of them. Hence,

$$
c_{n}(A) \simeq \sum_{i}\left\{m_{\lambda} d_{\lambda} \mid \lambda \in \operatorname{Par}(n) \cap C_{i}^{\prime}\right\} .
$$

If $1_{k, \ell}$ is not a basis element of any such $C_{i}^{\prime}$, then by Corollary 17 the sum over $C_{i}^{\prime}$ is at most $C n^{t}(\ell-\epsilon)^{n}$ for appropriate constants. But the Giambruno and Zaicev lemma shows that $c_{n}(A)$ is bounded below by a function of the form $C n^{t} \ell^{n}$ and so the sum of over the $C_{i}^{\prime}$ not containing $1_{k, \ell}$ may be ignored.

\section{The SUMS $\sum m_{\lambda} d_{\lambda}$ IN CONES}

In this section we fix an affine simplicial cone $\bar{C}=C\left(v_{0} ; v_{1}, \ldots, v_{r}\right) \subset \mathbb{R}^{k}$ whose basis vectors are partitions contained in $\mathbb{R}^{k, \ell}$ and the first one is $v_{1}=1_{k, \ell}$, a lattice $\underline{i}+(d \mathbb{Z})^{\ell}$, and a non-negative polynomial function with rational coefficients, $m_{\lambda}$. It is our goal to estimate

$$
\sum\left\{d_{\lambda} m_{\lambda} \mid \lambda \in \bar{C} \cap\left(\underline{i}+(d \mathbb{Z})^{\ell}\right) \cap \operatorname{Par}(n)\right\} .
$$

For convenience we modify this slightly by letting $C$ be the corresponding linear cone $C=C\left(v_{1}, \ldots, v_{r}\right)$ and replacing $\underline{i}$ by $\underline{i}-v_{0}$. This changes the sum to

$$
\sum\left\{d_{\lambda+v_{0}} m_{\lambda+v_{0}} \mid \lambda \in C \cap\left(\underline{i}+(d \mathbb{Z})^{\ell}\right) \cap \operatorname{Par}\left(n-\left|v_{0}\right|\right)\right\} .
$$

Our computation of this sum will imitate the computations in sections 1 and 2 of [14]. In that paper the asymptotic behavior of $\sum d_{\lambda}^{\beta}$ is computed, where $\lambda$ was restricted to have at most $\ell$ non-zero parts. In adapting the proof to the current situation we need to deal with the facts that our $\lambda$ is additionally restricted to be in an affine cone and a lattice, and that the sum is of a polynomial times $d_{\lambda}$ instead of a power of $d_{\lambda}$.

It is possible that some $\lambda_{i}=\lambda_{j}$ for every $\lambda \in C$. As in [14, we define $\theta_{1}, \ldots, \theta_{p}$ to be maximal such that

$$
\lambda_{1}=\cdots=\lambda_{\theta_{1}}, \lambda_{\theta_{1}+1}=\cdots=\lambda_{\theta_{1}+\theta_{2}}, \cdots \text { for all } \lambda \in C .
$$

Remark 20. Note that the sum $\sum m_{\lambda} d_{\lambda}$ with the above restrictions on $\lambda$ will be empty unless the sum of the parts of $\underline{i}$ is congruent to $n \bmod d$, which we take to be the case.

Generalizing the notation $\Lambda_{\ell}(n)$ from [14] we define $\Lambda_{\ell}^{\theta}(n)$ to be the set of all partitions of $n$ with height at most $\ell$ that satisfy equation (44). The degrees $d_{\lambda}$ of such partitions are estimated in [14, and we now recall some notation from that paper.

Definition 21. Given a partition $\lambda=\left(\lambda_{1}, \ldots, \lambda_{\ell}\right)$ of $n$, define $c_{i}$ by $\lambda_{i}=\frac{n}{\ell}+c_{i} \sqrt{n}$, $i=1, \ldots, \ell$. Let $\bar{D}(c)=\prod\left\{\left(c_{i}-c_{j}\right) \mid i<j, c_{i} \neq c_{j}\right\}, E(c)=\prod\{(i-j) \mid j<$ $\left.i, c_{i}=c_{j}\right\}$, and $\gamma_{\ell}=(2 \pi)^{-\frac{\ell-1}{2}} \ell^{\frac{1}{2} \ell^{2}}$.

Let $\lambda \in \Lambda_{\ell}^{\theta}(n)$ with each $\lambda_{\theta_{1}+\cdots+\theta_{i}} \neq \lambda_{\theta_{1}+\cdots+\theta_{i}+1}$. Then

$$
\bar{D}(c)=D^{\theta}(c)=\prod\left\{\left(c_{\theta_{1}+\cdots+\theta_{i}}-c_{\theta_{1}+\cdots+\theta_{j}}\right) \mid i<j\right\}
$$

and $E(c)=\left(\theta_{1}-1\right) ! \cdots\left(\theta_{p}-1\right)$ ! 
Lemma 22 (= Generalization 1.2 of [14). With notation as in the previous definition, assume that $\lambda \in \Lambda_{\ell}^{\theta}(n)$ is such that each $\left|c_{i}\right| \leq a$ and that each $c_{\theta_{1}+\cdots+\theta_{i}}-$ $c_{\theta_{1}+\cdots+\theta_{i+1}}$ is greater than or equal to some fixed $\delta>0$. Then

$$
d_{\lambda} \simeq \gamma_{\lambda} D^{\theta}(c)\left(\prod\left(\theta_{i}-1\right) !\right) e^{-\frac{\ell}{2} c^{2}} n^{-\frac{\ell^{2}-1}{2}} \sqrt{n}^{\left(\begin{array}{l}
\ell \\
2
\end{array}\right)-\sum_{i}\left(\begin{array}{c}
\theta_{i} \\
2
\end{array}\right)} \ell^{n},
$$

where by $c^{2}$ we mean $c_{1}^{2}+\cdots+c_{\ell}^{2}$.

In order to take the $v_{0}$ into account we use this lemma.

Lemma 23. Let $1 \leq \ell \leq k$, let $\nu$ be a fixed partition of height $k$ and denote by $\tilde{\nu}$ the sum $\sum_{i>\ell} \nu_{i}$. Let $\lambda \in \Lambda_{\ell}^{\theta}(n)$ be such that each $\lambda_{\theta_{1}+\cdots+\theta_{i}}-\lambda_{\theta_{1}+\cdots+\theta_{i}+1}$ goes to infinity. As $n$ goes to infinity,

$$
\frac{d_{\lambda+\nu}}{d_{\lambda}} \simeq b n^{\tilde{\nu}}
$$

where $b \in \mathbb{Q}$ is a constant.

Proof. By the Young-Frobenius formula (or by the hook formula),

$$
d_{\lambda}=\frac{|\lambda| !}{\prod_{i=1}^{k}\left(\lambda_{i}+k-i\right) !} \prod_{1 \leq i<j \leq k} \lambda_{i}-\lambda_{j}+j-i
$$

and similarly

$$
d_{\lambda+\nu}=\frac{|\lambda+\nu| !}{\prod_{i=1}^{k}\left(\lambda_{i}+\nu_{i}+k-i\right) !} \prod_{1 \leq i<j \leq k} \lambda_{i}+\nu_{i}-\lambda_{j}-\nu_{j}+j-i .
$$

Compare corresponding terms in the ratio $d_{\lambda+\nu} / d_{\lambda}$. The fraction $|\lambda+\nu| ! /|\lambda| !$ is asymptotic to $n^{|\nu|}$; for $i=1, \ldots, \ell$ the fraction $\left(\lambda_{i}+k-i\right) ! /\left(\lambda_{i}+\nu_{i}+k-i\right)$ ! is asymptotic to $\lambda_{i}^{-\nu_{i}}$; and for $i>\ell$ it is the rational number $(k-i) ! /\left(\nu_{i}+k-i\right)$ ! (independent of $\lambda$ ). Since $\left|c_{i}\right|<a, \lambda_{i} \simeq n / \ell, i=1, \ldots, \ell$, so

$$
\lambda_{1}^{-\nu_{1}} \cdots \lambda_{\ell}^{-\nu_{\ell}} \simeq\left(\frac{\ell}{n}\right)^{\nu_{1}+\cdots+\nu_{\ell}}
$$

Multiplying by $n^{|\nu|}$ gives a rational number times $n^{\tilde{\nu}}$. Finally, estimate

$$
A:=\prod_{1 \leq i<j \leq k} \frac{\lambda_{i}+\nu_{i}-\lambda_{j}-\nu_{j}+j-i}{\lambda_{i}-\lambda_{j}+j-i} .
$$

If $i \leq \ell$, then $\lambda_{i}-\lambda_{j} \rightarrow \infty$; hence

$$
\frac{\lambda_{i}+\nu_{i}-\lambda_{j}-\nu_{j}+j-i}{\lambda_{i}-\lambda_{j}+j-i} \simeq 1
$$

If $i>\ell$, then $\lambda_{i}=\lambda_{j}=0$, and the corresponding term is a rational number. It follows that $A \simeq r$ for some $r \in \mathbb{Q}$. The lemma now follows.

We now recall and modify some more definitions from [14].

Definition 24. Let $\Lambda_{\ell}^{\theta}(n, a)$ be the partitions in $\Lambda_{\ell}^{\theta}(n)$ with each $\left|c_{i}\right| \leq a$ and $\Lambda_{\ell}^{\theta}(n, a, \delta)$ be the partitions in $\Lambda_{\ell}^{\theta}(n, a)$ with each $c_{\theta_{1}+\cdots+\theta_{i}}-c_{\theta_{1}+\cdots+\theta_{i}+1} \geq \delta$. 
Combining this definition with the previous two lemmas yields:

Corollary 25. If $\lambda \in \Lambda_{\ell}^{\theta}(n, a, \delta)$, then

$$
d_{\lambda+v_{0}} \simeq K D^{\theta}(c) e^{-\frac{\ell}{2} c^{2}} n^{\tilde{v}_{0}-\frac{\ell^{2}-1}{2}} \sqrt{n}^{\left(\begin{array}{l}
\ell \\
2
\end{array}\right)-\sum_{i}\left(\begin{array}{c}
\theta_{i} \\
2
\end{array}\right)} \ell^{n}
$$

where $K$ is a constant in $\mathbb{Q}(\sqrt{\pi})$ depending only on $\ell, \theta$ and $v_{0}$.

Recall that $C$ is the simplicial cone $C=\left(v_{1}, \ldots, v_{r}\right)$ where $v_{1}=1_{k, \ell}$. Since $v_{1}, \ldots, v_{r}$ are linearly independent and by Lemma 18 they belong to $\mathbb{R}^{k, \ell}, r$ will be less than or equal to $\ell=\operatorname{dim} \mathbb{R}^{k, \ell}$, since elements of $\mathbb{R}^{k, \ell}$ have $k$ coordinates, but the last $k-\ell$ coordinates are $=0$.

Let $\lambda \in C$, so it is a positive combination of the $v_{i}$ 's, and write that combination in the form

$$
\lambda=\left(\frac{n}{\ell}+\alpha_{1} \sqrt{n}\right) v_{1}+\alpha_{2} \sqrt{n} v_{2}+\cdots+\alpha_{r} \sqrt{n} v_{r} .
$$

In order for the sum to be in $C$ we require that $\alpha_{i} \geq 0$ for $i=2, \ldots, r$ and $\frac{n}{\ell}+\alpha_{1} \sqrt{n} \geq 0$. Given the partition $\lambda=\left(\lambda_{1}, \ldots, \lambda_{\ell}\right)$, write $\lambda_{i}=\frac{n}{\ell}+c_{i} \sqrt{n}$. Comparing to the previous description we have

$$
\left(\frac{n}{\ell}+\alpha_{1} \sqrt{n}\right) v_{1}+\alpha_{2} \sqrt{n} v_{2}+\cdots+\alpha_{r} \sqrt{n} v_{r}=\left(\frac{n}{\ell}+c_{1} \sqrt{n}, \ldots, \frac{n}{\ell}+c_{r} \sqrt{n}\right) .
$$

Keeping in mind that $v_{1}=1_{k, \ell}$, this implies that

$$
\alpha_{1} v_{1}+\cdots+\alpha_{r} v_{r}=\left(c_{1}, \ldots, c_{\ell}\right)
$$

and so we define

$$
T\left(\alpha_{1}, \ldots, \alpha_{r}\right)=\sum_{i=1}^{r} \alpha_{i} v_{i}=\left(c_{1}, \ldots, c_{\ell}\right) .
$$

Let $\tau_{n}$ be given by

$$
\tau_{n}(\alpha)=\frac{n}{\ell} v_{1}+\sqrt{n} T(\alpha)=\frac{n}{\ell} v_{1}+\sqrt{n} \sum_{i=1}^{r} \alpha_{i} v_{i} .
$$

Since $C\left(v_{1}, \ldots, v_{r}\right)$ is assumed to be simplicial, the $v_{i}$ are linearly independent and so $T$ and hence $\tau_{n}$ are one-to-one. Note that $\left|v_{1}\right|=\ell$ and $\tau_{n}(\alpha)$ will have parts sum to $n$ precisely when $\sum \alpha_{i}\left|v_{i}\right|=0$, where by $\left|v_{i}\right|$ we mean the sum of the parts of $v_{i}$. This is equivalent to

$$
\alpha_{1}=-\sum_{i=2}^{r} \frac{\left|v_{i}\right|}{\ell} \alpha_{i} .
$$

Definition 26. Define $Q(a) \subseteq\left(\mathbb{R}^{+}\right)^{r-1}$ as follows. Let $\left(x_{2}, \ldots, x_{r}\right) \in\left(\mathbb{R}^{+}\right)^{r-1}$ and denote $x_{1}=x_{1}(x)=-\sum_{i=2}^{r} \frac{\left|v_{i}\right|}{\ell} x_{i}$. By (5), $T\left(x_{1}(x), x\right)=T\left(x_{1}, x_{2}, \ldots, x_{r}\right)=$ $\left(c_{1}, \ldots, c_{\ell}\right)$. Given $a>0$, define $Q(a)$ via: $x=\left(x_{2}, \ldots, x_{r}\right) \in Q(a)$ iff $T\left(x_{1}(x), x\right)=$ $\left(c_{1}, \ldots, c_{\ell}\right)$ and all $\left|c_{i}\right| \leq a$. Continuing in this vein, note that $c_{1}=\cdots=$ $c_{\theta_{1}}, \quad c_{\theta_{1}+1}=\cdots=c_{\theta_{1}+\theta_{2}}, \ldots$, in the image of $T$ and so define $Q(a, \delta)$ to be the subset of $Q(a)$ with the additional conditions $c_{\theta_{1}+\cdots+\theta_{i}}-c_{\theta_{1}+\cdots+\theta_{i}+1} \geq \delta$.

Remark 27. Note that both $Q(a)$ and $Q\left(a, a^{-r}\right)$ tend to $\left(\mathbb{R}^{+}\right)^{r-1}$ as $a \rightarrow \infty$.

Definition 28. Let

$$
S_{\bar{C}}(n)=\sum\left\{m_{v_{0}+\lambda} d_{v_{0}+\lambda} \mid \lambda \in \operatorname{Par}(n) \cap C \cap\left(\underline{i}+(d \mathbb{Z})^{\ell}\right)\right\} .
$$


Two related sums are

$$
S_{\bar{C}}(n, a, \theta)=\sum\left\{m_{v_{0}+\lambda} d_{v_{0}+\lambda} \mid \lambda \in \Lambda_{\ell}^{\theta}(n, a) \cap C \cap\left(\underline{i}+(d \mathbb{Z})^{\ell}\right)\right\}
$$

and

$$
S_{\bar{C}}(n, a, \delta, \theta)=\sum\left\{m_{v_{0}+\lambda} d_{v_{0}+\lambda} \mid \lambda \in \Lambda_{\ell}^{\theta}(n, a, \delta, \theta) \cap C \cap\left(\underline{i}+(d \mathbb{Z})^{\ell}\right)\right\} .
$$

Remark 29. Note by equation (3) that $S_{C}(n)$ represents the contribution of the characters in the cone $C$ intersecting the lattice $\underline{i}+(d \mathbb{Z})^{\ell}$ to the codimension, and recall by $\operatorname{Remark} 20$ that $\operatorname{Par}(n) \cap\left(\underline{i}+(d \mathbb{Z})^{\ell}\right)$ will be empty unless $n \equiv|i| \bmod d$. Hence, if $n \not \equiv|i| \bmod d$, each of $S_{\bar{C}}(n), S_{\bar{C}}(n, a)$, etc. equals zero.

We note that $m_{\lambda}$ is assumed to be a non-negative polynomial function of $\lambda_{1}, \ldots$, $\lambda_{\ell}$ with rational coefficients. Let the vector $v_{i}$ have coordinates $v_{i j}$. Then it follows from the definition of $\tau_{n}$ that if $\lambda=\tau_{n}(\alpha)$, then each $\lambda_{j}$ equals $v_{1 j} \frac{n}{\ell}+\sqrt{n} \sum_{i} \alpha_{i} v_{i j}$. Let the multiplicity function $m_{\lambda}$ be equal to the polynomial $m\left(x_{1}, \ldots, x_{\ell}\right)$ on the intersection of the cone and the lattice. By substitution,

$$
m_{v_{0}+\lambda}=m\left(v_{0}+\frac{n}{\ell} v_{1}+\sqrt{n} \sum \alpha_{i} v_{i}\right),
$$

and so each $x_{j}$ is replaced by $v_{0 j}+v_{1 j} \frac{n}{\ell}+\sum_{i} \alpha_{i} v_{i j}$ and so

$$
m_{v_{0}+\lambda} \simeq n^{\mu} g\left(\alpha_{1}, \ldots, \alpha_{r}\right),
$$

where $\mu$ is an integer or a half-integer and $g$ is a polynomial with rational coefficients. The following is a direct analogue of Lemma 2.2 of [14] and the proof is the same.

Lemma 30. Referring to Remarks 20 and 29 , let $n \equiv|\underline{i}| \bmod d$. Then, as $n \rightarrow \infty$ (namely, $n=|\underline{i}|+q \cdot d$ and $q \rightarrow \infty$ ), the sum $S_{\bar{C}}(n, a, \delta, \theta)$ is asymptotic to

$$
\sum n^{\mu} f(\alpha) e^{-\frac{\ell}{2} T(\alpha)^{2}} n^{\tilde{\nu}_{0}-\frac{\ell^{2}-1}{2}} \sqrt{n}^{\left(\begin{array}{l}
\ell \\
2
\end{array}\right)-\sum_{j}\left(\begin{array}{c}
\theta_{j} \\
2
\end{array}\right)} \ell^{n+\left|v_{0}\right|},
$$

where the sum is over $\left(\alpha_{2}, \ldots, \alpha_{r}\right) \in Q(a, \delta), \alpha_{1}=-\sum \frac{\left|v_{i}\right|}{\ell} \alpha_{i}$ such that $\tau_{n}(\alpha) \in$ $\underline{i}+(d \mathbb{Z})^{\ell}$, and where $f(\alpha)=g(\alpha) K D^{\theta}(T(\alpha))$.

Note that $Q(a)$ and $Q(a, \delta)$ are bounded $(r-1)$-dimensional surfaces and we can consider their volumes. The proofs of Lemmas 2.3 and 2.4 of [14] now go over essentially unchanged.

Lemma 31 (= Lemma 2.4 of [14]). If $\delta=\frac{1}{a^{r}}$, then

$$
\lim _{a \rightarrow \infty} \operatorname{vol}_{r-1}(Q(n, a)-Q(n, a, \delta))=0 .
$$

Following 14] we let $D_{\delta}(c)$ equal $\prod\left\{\left(c_{i}-c_{j}\right) \mid i<j, c_{i}-c_{j} \geq \delta\right\}$.

We now record the analogues of the next three lemmas of [14. The proofs are essentially unchanged, although since we are restricting to partitions in the intersection of the cone $C$ with the lattice $\left(\underline{i}=(d \mathbb{Z})^{\ell}\right)$ we replace the condition that $\frac{n}{\ell}+c_{j} \sqrt{n} \geq 0$ must be an integer with $\tau_{n}(\alpha) \in\left(\underline{i}+(d \mathbb{Z})^{\ell}\right)$ and, in order for the intersection to be non-empty, we require $n \equiv|\underline{i}| \bmod d$ as in the previous lemma.

Lemma 32 (= Proposition 2.5 of [14]). Let $n \equiv|\underline{i}| \bmod d$ and let

$$
u(n, a)=\sum g(\alpha) D_{a^{-r}}(T(\alpha)) e^{-\frac{\ell}{2}\left(T(\alpha)^{2}\right)}
$$


be summed over $\left(\alpha_{2}, \ldots, \alpha_{r}\right) \in Q(n, a)-Q\left(n, a, a^{-r}\right)$ and $\alpha_{1}=-\sum \frac{\left|v_{i}\right|}{\ell} \alpha_{i}$ such that $\tau_{n}(\alpha) \in\left(\underline{i}+(d \mathbb{Z})^{\ell}\right)$. As $n \rightarrow \infty$ (namely, $n=|\underline{j}|+q \cdot d$ and $\left.q \rightarrow \infty\right)$,

$$
\lim _{a \rightarrow \infty} \lim _{n \rightarrow \infty} \frac{u(n, a)}{\sqrt{n}^{r-1}}=0 .
$$

Now, we may choose a small cube $U$ in $Q(1)$ with volume $V_{1}$ such that

$$
g(\alpha) D_{a^{-r}}(T(\alpha)) e^{-\frac{\ell}{2}\left(T(\alpha)^{2}\right)} \geq m>0 \text { on } U .
$$

We now have this analogue of Lemmas 2.6 and 2.7 of 14 .

Lemma 33 (= Lemma 2.6 of [14]). If $a \geq 1$ and $a$ is so large that $Q\left(a, a^{-r}\right)$ contains $U$, then $\lim _{n \rightarrow \infty} \frac{v(n, a)}{\sqrt{n}^{r-1}} \geq m V_{1}$, where

$$
v(n, a)=\sum g(\alpha) D_{a^{-r}}(T(\alpha)) e^{-\frac{\ell}{2}\left(T(\alpha)^{2}\right)}
$$

summed over $\left(\alpha_{2}, \ldots, \alpha_{r}\right) \in Q\left(n, a, a^{-r}\right)$ and $\alpha_{1}=-\sum \frac{\left|v_{i}\right|}{\ell} \alpha_{i}$ such that $\tau_{n}(\alpha) \in$ $\left(\underline{i}+(d \mathbb{Z})^{\ell}\right)$.

Lemma 34 (= Lemma 2.7 of [14]). Let

$$
w(n, a)=g(\alpha) D_{\delta}(T(\alpha)) e^{-\frac{\ell^{3}}{3}\left(T(\alpha)^{2}\right)}
$$

be summed over $\left(\alpha_{2}, \ldots, \alpha_{r}\right) \notin Q(n, a)$ and $\alpha_{1}=-\sum \frac{\left|v_{i}\right|}{\ell} \alpha_{i}$ such that $\tau_{n}(\alpha) \in$ $\left(\underline{i}+(d \mathbb{Z})^{\ell}\right)$. Then

$$
\lim _{a \rightarrow \infty} \lim _{n \rightarrow \infty} \frac{w(n, a)}{\sqrt{n}^{r-1}}=0 .
$$

Just as in 14 we can combine these lemmas together with Lemma 1.6 of that work to get the following analogue of Theorem 2.8 of [10]:

Theorem 35. Let $n \equiv|\underline{i}| \bmod d$, as above, and let $\sigma(n, a)=\frac{S_{\bar{C}}(n)}{S_{\bar{C}}\left(n, a, a^{-r}\right)}$. Then, as $n \rightarrow \infty$ (namely, $n=|\underline{j}|+q \cdot d$ and $q \rightarrow \infty), \lim _{a \rightarrow \infty} \lim _{n \rightarrow \infty} \sigma(n, a)=1$.

As in [14, the limit of $\frac{v(n, a)}{\sqrt{n}^{r-1}}$, defined in Lemma 33, converges to a certain integral. To see this, we need some concepts from lattices. The volume of a lattice

$$
\mathcal{L}=b_{0}+b_{a} \mathbb{Z}+\cdots+b_{n} \mathbb{Z} \subset \mathbb{R}^{n}
$$

is defined to be the volume of the parallelepiped

$$
\operatorname{vol}(\mathcal{L})=\operatorname{vol}\left\{x_{1} b_{1}+\cdots+x_{n} b_{n} \mid 0 \leq x_{i} \leq 1, i=1, \ldots, n\right\} .
$$

Now let $D \subseteq \mathbb{R}^{n}$ be a "nice" domain, $f: D \rightarrow \mathbb{R}$ a continuous function, and $\mathcal{L}$ an $n$-dimensional lattice with small volume. Then

$$
\sum_{x \in \mathcal{L} \cap D} f(x) \approx \frac{1}{v} \int_{R} f(x) d x
$$

and the two sides become equal as $\operatorname{vol}(\mathcal{S}) \rightarrow 0$.

To apply this theory to our case, we start with the lattice

$$
\mathcal{L}_{0}=\left\{\left(\alpha_{2}, \ldots, \alpha_{r}\right) \mid \alpha_{1} v_{1}+\cdots+\alpha_{r} v_{r} \in(d \mathbb{Z})^{\ell}\right\},
$$

where, as usual, $\alpha_{1}=-\sum \frac{\left|\alpha_{i}\right|}{\ell}$. Let $\operatorname{vol}\left(\mathcal{L}_{0}\right)=V$. Next define

$$
\mathcal{L}=\left\{\left(\alpha_{2}, \ldots, \alpha_{r}\right) \mid \tau_{n}(\alpha) \in \vec{v}+(d \mathbb{Z})^{\ell}\right\} .
$$


Then $\operatorname{vol}(\mathcal{L})=\frac{V}{\sqrt{n}^{r-1}}$ and so $\lim _{n \rightarrow \infty} \frac{v(n, a)}{\sqrt{n}^{r-1}}$ converges to the integral

$$
\frac{1}{V} \int \cdots \int_{Q\left(a, a^{-r}\right)} g\left(x_{1}, \ldots, x_{r}\right) D^{\theta}\left(T\left(x_{1}, \ldots, x_{r}\right)\right) e^{-\frac{\ell}{2} T\left(x_{1}, \ldots, x_{r}\right)^{2}} d x_{2} \cdots d x_{r},
$$

where $x_{1}=-\sum \frac{\left|v_{i}\right|}{\ell} x_{i}$. Taking the limit as $a \rightarrow \infty$ gives the integral

$$
I(C)=\frac{1}{V} \int_{0}^{\infty} \cdots \int_{0}^{\infty} g\left(x_{1}, \ldots, x_{r}\right) D^{\theta}\left(T\left(x_{1}, \ldots, x_{r}\right)\right) e^{-\frac{\ell}{2} T\left(x_{1}, \ldots, x_{r}\right)^{2}} d x_{2} \cdots d x_{r} .
$$

Then, analogously to Theorem 2.10 of [14], we have

Theorem 36. Let $n \equiv|\underline{i}| \bmod d$, as above. Then, as $n \rightarrow \infty$,

$$
S_{\bar{C}}(n) \simeq I(C) K \sqrt{n}^{\left(\begin{array}{l}
\ell \\
2
\end{array}\right)-\sum_{i}\left(\begin{array}{c}
\theta_{i} \\
2
\end{array}\right)+r-1} n^{\tilde{v}_{0}-\frac{t^{2}-1}{2}} \ell^{n} .
$$

In particular, for these values of $n, S_{\bar{C}}(n)$ is asymptotic to a constant times $n$ to the power of an integer or half-integer times a positive integer to the power of $n$.

In order to get more information about the constant factor, we would need to know more about the integral $I(C)$. We close this section with a conjecture.

Conjecture. The integral $I(C)$ is in $\mathbb{Q}\left[\sqrt{a_{1}}, \ldots, \sqrt{a_{n}}, \sqrt{2 \pi}\right]$ for some integers $a_{1}$, $\ldots, a_{n}$, under the hypothesis that $g$ is a polynomial with rational coefficients and that the vectors $v_{i}$ used to define $T$ and $\theta$ have rational coordinates.

\section{LAST STEPS OF THE PROOF}

In the previous section, we estimated $\sum m_{\lambda} d_{\lambda}$ for $\lambda$ in a simplicial cone $C$, assuming $m_{\lambda}$ to be polynomial. In general, $c_{n}(A)$ will be a sum of such and we now deal with the problem of taking the sum.

Definition 37. Let $w_{1}+C_{1}, \ldots, w_{a}+C_{a}$ be rational affine simplicial cones as in Theorem [19, I.e., it is a maximal list such that each $C_{j}$ contains $1_{k, \ell}$ as a basis element and $m_{\lambda}$ is almost polynomial on each $C_{j}$; see Definition 6. By Theorem 19 those cones not containing $1_{k, \ell}$ in their basis don't contribute to the asymptotics of $c_{n}(A)$ and so we may disregard them.

Let $d$ be a modulus such that $m_{\lambda}$ equals a polynomial on each $w_{j}+C_{j} \cap\left(\underline{i}+(d \mathbb{N})^{k}\right)$, for $\underline{i} \in(d \mathbb{N})^{k}$. Denote this polynomial by $m(\underline{i}, j)(\lambda)$. As in Remark 29 , this intersection of a cone and a lattice will contain partitions of $n$ if and only if $n \equiv|\underline{i}|$ $(\bmod d)$. Denote by $\mathcal{S}(n)$ the set of all $\underline{i} \in\left(\mathbb{Z}_{d}\right)^{k}$ and $1 \leq j \leq a$ such that

$$
\operatorname{Par}(n) \cap\left(\underline{i}+(d \mathbb{Z})^{k}\right) \cap C_{j} \neq 0 .
$$

Now, using this notation,

$c_{n}(A) \simeq \sum_{(\underline{i}, j) \in \mathcal{S}(n)} \sum\left\{m_{\lambda} d_{\lambda} \mid \lambda \in \operatorname{Par}(n) \cap\left(\underline{i}+(d \mathbb{Z})^{k}\right) \cap C_{j}\right\}=\sum_{(\underline{i}, j) \in \mathcal{S}(n)} S_{C_{j}}\left(n-\left|w_{j}\right|\right)$.

Combining this with Theorem 36 now gives

Lemma 38. Let $d$ be as in Definition 37 so that $m_{\lambda}$ is a polynomial on each $\left(\underline{i}+(d \mathbb{Z})^{k}\right) \cap C_{j}$ where $1 \leq j \leq a$ and $\underline{i} \in\{0, \ldots, d-1\}^{k}$. Let $0 \leq m \leq d-1$, and let $n \equiv m(\bmod d)$ go to infinity, i.e., $n=q k+m$ and $q \rightarrow \infty$. Then there are constants $a_{m}$ and $t_{m}$, the latter in $\frac{1}{2} \mathbb{Z}$, such that, for these $n$, the codimension sequence of $A$ satisfies $c_{n}(A) \simeq a_{m} n^{t_{m}} \ell^{n}$. 
In order to prove that the $t_{m}$ are equal we need only assume that the $c_{n}$ are non-decreasing. This is true whenever the algebra $A$ has 1 , but it is also true under a weaker hypothesis, such as $x A \neq 0$ for every $0 \neq x \in A$.

Lemma 39. With notation as in the previous lemma, if $x A \neq 0$ for all $0 \neq x \in A$, then all $t_{m}$ are equal.

Proof. The map $f\left(x_{1}, \ldots, x_{n}\right) \mapsto f\left(x_{1}, \ldots, x_{n}\right) x_{n+1}$ takes non-identities of $A$ to non-identities. It follows that the codimension sequence is non-decreasing. If not all $t_{m}$ were equal, there would be an $m$ such that, say, $t_{m}>t_{m+1}$. But this would imply that for all $n \equiv m \bmod d$,

$$
\frac{c_{n+1}(A)}{c_{n}(A)} \simeq \frac{a_{m+1}(n+1)^{t_{m+1}} \ell^{n+1}}{a_{m} n^{t_{m}} \ell^{n}} \longrightarrow 0,
$$

contradicting the fact that the $c_{n}(A)$ are non-decreasing.

In this case we have

$$
a_{1} n^{t} \ell^{n} \leq c_{n}(A) \leq a_{2} n^{t} \ell^{n}
$$

for some $0<a_{1} \leq a_{2}$

Drensky showed in 8 that the cocharacter sequence of $A$ is Young derived, whenever $A$ has a unit. We record that theorem by way of reminding the reader of the definition of Young derived sequences.

Theorem 40. Let $A$ be a $p$. i. algebra with unit. Then there exists a sequence of $S_{n}$ characters $\left\{\psi_{n}\right\}_{n=0}^{\infty}$ such that for all $n, \chi_{n}(A)=\sum \chi^{(n-i)} \hat{\otimes} \psi_{i}$. Let each $\chi_{n}(A)=\sum m_{\lambda} \chi^{\lambda}$ and each $\psi_{n}=\sum m_{\lambda}^{\prime} \chi^{\lambda}$. Then, using Young's rule, the relation between $\chi(A)$ and $\{\psi\}$ can be restated as

$$
m_{\lambda}=\sum\left\{m_{\nu}^{\prime} \mid \nu \subseteq \lambda, \lambda / \nu \text { a horizontal strip }\right\} .
$$

Letting $c_{n}^{\prime}(A)$ be the degree of $\psi_{n}$ we get

$$
c_{n}(A)=\sum_{i=0}^{n}\left(\begin{array}{l}
n \\
i
\end{array}\right) c_{i}^{\prime}
$$

We now need to verify that $c_{n}^{\prime}(A)$ has growth properties similar to those of $c_{n}(A)$ as described in Theorem 36. This will be Lemma 43. In order to prove this, we need to show that the sequence $\left\{\psi_{n}\right\}$ has the same properties of $\chi_{n}(A)$ that we used in the proof of Theorem 36. First note that $\left\{\psi_{n}\right\}$ is supported by partitions of height at most $k$, since the cocharacter sequence of $A$ is. The next ingredient we need is Theorem 5. This theorem is a consequence of the fact that the Poincaré series for (the generic algebra of) $A$ is a nice rational function, see [5, which is based on [3]. A nice rational function is one in which the numerator and denominator are polynomials with integer coefficients and the denominator can be written as a product of terms of the form $(1-u)$, where $u$ is a monic monomial. Section 2 of [5] shows that if a nice rational function is also a symmetric function, then the coefficients of the Schur functions are as in Theorem 5. Let $m_{\lambda}^{\prime}$ denote the multiplicity of $\chi^{\lambda}$ in $\left\{\psi_{n}\right\}$, so $\psi_{n}=\sum m_{\lambda}^{\prime} \chi^{\lambda}$. Define $g\left(x_{1}, \ldots, x_{k}\right)$ to be $\sum m_{\lambda}^{\prime} S_{\lambda}\left(x_{1}, \ldots, x_{k}\right)$, where $S_{\lambda}\left(x_{1}, \ldots, x_{k}\right)$ are Schur functions. Then Drensky proved that $g$ is related to the Poincaré series of $A$ via

$$
P_{k}(A)=\frac{g\left(x_{1}, \ldots, x_{k}\right)}{\left(1-x_{1}\right) \cdots\left(1-x_{k}\right)} .
$$


Since $P_{k}(A)$ is a nice rational function, $g(x)$ will be one also, and it follows from the techniques of [5] that the $m_{\lambda}^{\prime}$ satisfy the conclusion of Theorem 5 .

Lemma 41. Let $m_{\lambda}^{\prime}$ be as above. Then there exists an integer $d$ and a partition of $\left(\mathbb{R}^{+}\right)^{k}$ into regions $R_{1}, \ldots, R_{m}$, each a finite intersection of rational affine linear cones such that $m_{\lambda}^{\prime}$ is given by a polynomial on each $R_{a} \cap\left(\underline{i}+(d \mathbb{Z})^{k}\right)$, where $\underline{i} \in$ $\{0, \ldots, d-1\}^{k}$.

We now prove these analogues of results that Giambruno and Zaicev proved about the cocharacter sequence of $A$; see Lemma 18 and the paragraph preceding it.

Lemma 42. For $e(A)=\ell$, the multiplicities $m_{\lambda}^{\prime}$ are zero for partitions with $\lambda_{\ell}$ large, and, for every $n$, there are non-zero $m_{\lambda}^{\prime}$ with $\lambda$ (a partition of $n \cdot(\ell-1)$ ) close to the rectangle of height $\left(n^{\ell-1}\right)$.

Proof. Theorem 40 says that $m_{\lambda}$ is the sum of all $m_{\nu}^{\prime}$ such that $\lambda / \nu$ is a horizontal strip. This condition is equivalent to $\lambda_{i+1} \leq \nu_{i} \leq \lambda_{i}$ for all $i$. Now if $\nu=\left(\nu_{1}, \ldots, \nu_{k}\right)$ is a partition of height greater than or equal to $\ell$ with $m_{\nu}^{\prime}>0$, let $\lambda$ be the partition obtained from $\nu$ by increasing $\nu_{\ell+1}$ to $\nu_{\ell}$. Then $\lambda / \nu$ is a horizontal strip and so $m_{\lambda} \geq m_{\nu}^{\prime}>0$. Since $e(A)=\ell$, there is a constant $K$ such that $\lambda_{\ell+1} \leq K$ whenever $m_{\lambda}>0$ (see after Corollary 17). Then the above implies that, also, $\nu_{\ell} \leq K$ if $m_{\nu}^{\prime}>0$.

Likewise, since $m_{\lambda}$ is non-zero for some $\lambda$ close to $\left(n^{\ell}\right)$, there must be a $\nu$ with $m_{\nu}^{\prime}$ non-zero and $\lambda / \nu$ a horizontal strip. Hence each $\nu_{i}-\nu_{i+1}$ is bounded, and by the first part of the lemma, $\nu_{\ell}$ is bounded. Hence, $\nu$ must be close to $n^{\ell-1}$.

The results of sections 1 and 2 now apply to $c_{n}^{\prime}$, yielding the following analogue of Lemma 38 ,

Lemma 43. Let $0 \leq m \leq d-1$ and let $n \equiv m(\bmod d)$ go to infinity. Then there are constants $b_{m}$ and $t_{m}^{\prime}$ with $t_{m}^{\prime} \in \frac{1}{2} \mathbb{Z}$ such that $c_{n}^{\prime} \simeq b_{m} n^{t_{m}^{\prime}}(\ell-1)^{n}$.

Note that the $c_{n}^{\prime}(A)$ need not be increasing, and so the $t_{m}^{\prime}$ need not be equal, so we cannot invoke an analogue of Lemma 39 in this case. For example, if $A$ is the infinite-dimensional Grassmann algebra, then $c_{n}^{\prime}(A)$ is 0 or 1 depending on whether $n$ is odd or even, respectively.

We now combine this lemma with equation (6) to prove that the $a_{m} n^{t_{m}}$ are all equal. It follows from that equation that

$$
c_{n}(A)=\sum_{q=0}^{n}\left(\begin{array}{l}
n \\
q
\end{array}\right) c_{q}^{\prime}=\sum_{m=0}^{d-1} \sum_{\substack{0 \leq q \leq n \\
q \equiv m(\bmod d)}}\left(\begin{array}{l}
n \\
q
\end{array}\right) c_{q}^{\prime} .
$$

Lemma 44. The codimensions $c_{n}(A)$ are asymptotic to

$$
\sum_{m=0}^{d-1} \sum_{\substack{0 \leq q \leq n \\
q \equiv m(\bmod d)}}\left(\begin{array}{l}
n \\
q
\end{array}\right) b_{m} \cdot q^{t_{m}^{\prime}}(\ell-1)^{q} .
$$

Proof. Use Lemma 43, Given $\epsilon>0$ there exists $q_{0}$ such that for $q>q_{0}$,

$$
(1-\epsilon) b_{m} \cdot q^{t_{m}^{\prime}}(\ell-1)^{q} \leq c_{q}^{\prime}(A) \leq(1+\epsilon) b_{m} \cdot q^{t_{m}^{\prime}}(\ell-1)^{q},
$$


where $q \equiv m \bmod d$, for any $m$. Now observe that $\sum_{q \leq q_{0}}\left(\begin{array}{l}n \\ q\end{array}\right) q^{t}(\ell-1)^{q}$ and $\sum_{q \leq q_{0}}\left(\begin{array}{l}n \\ q\end{array}\right) c_{q}^{\prime}(A)$ are both bounded by a polynomial in $n$ and so may be disregarded.

Lemma 45. For each $0 \leq m \leq d-1$, the sum $\sum\left(\begin{array}{l}n \\ q\end{array}\right) b_{m} \cdot q^{t_{m}^{\prime}}(\ell-1)^{q}$, summed over $0 \leq q \leq n, q \equiv m(\bmod d)$, is asymptotic to $a n^{t_{m}} \ell^{n}$, where a is a constant depending on $b_{m}, \ell$ and $t_{m}^{\prime}$. In particular the asymptotics do not depend on $n \bmod$ $d$.

Proof. Let $\omega$ be a primitive $d^{\text {th }}$ root of 1 and note that $\sum_{\alpha=0}^{d-1} \omega^{(q-m) \alpha}$ is zero if $q \not \equiv m(\bmod d)$ and is $d$ if $q \equiv m(\bmod d)$. So, the $\operatorname{sum} \sum\left(\begin{array}{l}n \\ q\end{array}\right) b_{m} \cdot q^{t_{m}^{\prime}}(\ell-1)^{q}$, summed over $0 \leq q \leq n, q \equiv m(\bmod d)$ can be rewritten as

$$
\frac{1}{d} \sum_{q=0}^{n} \sum_{\alpha=0}^{d-1} \omega^{(q-m) \alpha}\left(\begin{array}{l}
n \\
q
\end{array}\right) b_{m} q^{t_{m}^{\prime}}(\ell-1)^{q}=\frac{1}{d} \sum_{\alpha=0}^{d-1} \omega^{-m \alpha} \sum_{q=0}^{n}\left(\begin{array}{l}
n \\
q
\end{array}\right) b_{m} q^{t_{m}^{\prime}}\left(\omega^{\alpha}(\ell-1)\right)^{q} .
$$

Now, using Lemma 1.1 of [2] we get that the inner sum is asymptotic to a constant times

$$
\left.n^{t_{m}^{\prime}}\left(\omega^{\alpha}(\ell-1)+1\right)\right)^{n}
$$

The absolute value of $\left(\omega^{\alpha}(\ell-1)+1\right)$ is maximized when $\omega^{\alpha}(\ell-1)$ is real and positive, which happens precisely when $\alpha=0$, and so these terms dominate the sum. The lemma now follows.

Substituting Lemma 45 into Lemma 43 yields

$$
c_{n}(A) \simeq \sum_{m=0}^{d-1} a_{m} n^{t_{m}^{\prime}} \ell^{n}
$$

If $g$ is the maximum value of the $t_{m}^{\prime}$ and $a=\sum\left\{b_{m} \mid t_{m}^{\prime}=g\right\}$, then this sum is asymptotic to $a n^{g} \ell^{n}$ and $2 g$ is an integer.

\section{REFERENCES}

[1] W. Beckner and A. Regev, Asymptotics and algebraicity of some generating functions, Adv. Math. 65 (1987), 1-15. MR893467 (88h:05008)

[2] W. Beckner and A. Regev, Asymptotic estimates using probability, Adv. Math. 138 (1998), 1-14. MR1645060 (99j:05011)

[3] A. Ya. Belov, Rationality of Hilbert series with respect to free algebras, Russian Math. Surveys 52 (1997), 394-395. MR1480146 (98f:16018)

[4] A. Berele, Approximate multiplicities in the trace cocharacter sequence of two three-by-three matrices, Comm. Algebra 25 (1997) 1975-1983. MR1446144 (98j:05129)

[5] A. Berele, Applications of Belov's theorem to the cocharacter sequence of p. i. algebras, J. of Alg. 298 (2006), 208-214. MR2215124 (2006m:16023)

[6] A. Berele and A. Regev, Codimensions of products and of intersections of verbally prime T-ideals, Israel J. Math. 103 (1998), 17-28. MR.1613536 (99b:16037)

[7] A. Berele and A. Regev, Exponential growth for codimensions of some p. i. algebras, J. of Alg. 241 (2001), 118-145. MR.1838847 (2002k:16046)

[8] V. Drensky, Codimensions of T-ideals and Hilbert series of relatively free algebras, $J$. of Alg. 89 (1984), 178-223. MR765766 (86b:16010)

[9] V. Drensky and G. K. Genov, Multiplicities of Schur functions in invariants of two $3 \times 3$ matrices, J. Algebra 264 (2003), 496-519. MR1981418 (2004c:16037)

[10] A. Giambruno and M. V. Zaicev, On codimension growth of finitely generated associative algebras, Adv. Math. 140 (1998), 145-155. MR.1658530 (99k:16049)

[11] A. Giambruno and M. V. Zaicev, Exponential codimension growth of PI algebras: An exact estimate, Adv. Math. 142 (1999), 221-243. MR.1680198(2000a:16048) 
[12] B. Grünbaum, Convex Polytopes, Interscience, London, 1967. MR0226496 (37:2085)

[13] A. Guterman and A. Regev, On the growth of identities, In: Algebra (Moscow: 1998), 319330, de Gruyter, Berlin, 2000. MR 1754678(2001a:16039)

[14] A. Regev, Asymptotic values for degrees associated with strips of Young diagrams, Adv. in Math. 41 (1981), 115-136. MR625890 (82h:20015)

[15] A. Regev, Algebras satisfying a Capelli identity, Israel J. Math. 33 (1979), 149-154. MR571250 (81i:16022)

[16] G. M. Zeigler, Lectures on Polytopes, Graduate Texts in Mathematics 152, Springer-Verlag, New York, 1995. MR.1311028 (96a:52011)

Department of Mathematics, DePaul University, Chicago, Illinois 60614

E-mail address: aberele@condor.depaul.edu

Department of Theoretical Mathematics, Weizmann Institute, Rehovot, Israel

E-mail address: amitai.regev@wisdom.weizmann.ac.il 